\title{
SENTINEL-2 LEVEL 1 PRODUCTS AND IMAGE PROCESSING PERFORMANCES
}

\author{
S. J Baillarin ${ }^{\text {a, } *, \text { A. Meygret }}{ }^{\text {a }}$, C. Dechoz ${ }^{\text {a }}$, B. Petrucci ${ }^{\text {a }}$, S. Lacherade ${ }^{\text {a }}$, T. Tremas ${ }^{\text {a }}$, \\ C. Isola ${ }^{b}$, P. Martimort ${ }^{b}$, F. Spoto ${ }^{b}$ \\ ${ }^{a}$ CNES, 18, Avenue E. Belin, 31401 Toulouse Cedex 4, France - simon.baillarin@cnes.fr \\ ${ }^{\mathrm{b}}$ ESTEC, ESA, Keplerlaan 1, PO Box 299, 2200 AG Noordwijk ZH, The Netherlands
}

KEY WORDS: Radiometry, Geometry, Image processing, Performances, GMES, Sentinel-2

\begin{abstract}
:
In partnership with the European Commission and in the frame of the Global Monitoring for Environment and Security (GMES) program, the European Space Agency (ESA) is developing the Sentinel-2 optical imaging mission devoted to the operational monitoring of land and coastal areas.

The Sentinel-2 mission is based on a satellites constellation deployed in polar sun-synchronous orbit. While ensuring data continuity of former SPOT and LANDSAT multi-spectral missions, Sentinel-2 will also offer wide improvements such as a unique combination of global coverage with a wide field of view $(290 \mathrm{~km})$, a high revisit (5 days with two satellites), a high resolution $(10 \mathrm{~m}, 20 \mathrm{~m}$ and $60 \mathrm{~m})$ and multi-spectral imagery (13 spectral bands in visible and shortwave infra-red domains).

In this context, the Centre National d'Etudes Spatiales (CNES) supports ESA to define the system image products and to prototype the relevant image processing techniques.

This paper offers, first, an overview of the Sentinel-2 system and then, introduces the image products delivered by the ground processing: the Level-0 and Level-1A are system products which correspond to respectively raw compressed and uncompressed data (limited to internal calibration purposes), the Level-1B is the first public product: it comprises radiometric corrections (dark signal, pixels response non uniformity, crosstalk, defective pixels, restoration, and binning for $60 \mathrm{~m}$ bands); and an enhanced physical geometric model appended to the product but not applied, the Level-1C provides ortho-rectified top of atmosphere reflectance with a sub-pixel multi-spectral and multi-date registration; a cloud and land/water mask is associated to the product. Note that the cloud mask also provides an indication about cirrus. The ground sampling distance of Level-1C product will be $10 \mathrm{~m}, 20 \mathrm{~m}$ or $60 \mathrm{~m}$ according to the band. The final Level-1C product is tiled following a pre-defined grid of $100 \times 100 \mathrm{~km}^{2}$, based on UTM/WGS84 reference frame.

The stringent image quality requirements are also described, in particular the geo-location accuracy for both absolute (better than $12.5 \mathrm{~m}$ ) and multi-temporal (better than 0.3 pixels) cases.

Then, the prototyped image processing techniques (both radiometric and geometric) will be addressed.

The radiometric corrections will be first introduced. They consist mainly in dark signal and detector relative sensitivity correction, crosstalk correction and MTF restoration. Then, a special focus will be done on the geometric corrections. In particular the innovative method of automatic enhancement of the geometric physical model will be detailed. This method takes advantage of a Global Reference Image database, perfectly geo-referenced, to correct the physical geometric model of each image taken. The processing is based on an automatic image matching process which provides accurate ground control points between a given band of the image to refine and a reference image, allowing to dynamically calibrate the viewing model. The generation of the Global Reference Image database made of Sentinel-2 pre-calibrated mono-spectral images will be also addressed.
\end{abstract}

In order to perform independent validation of the prototyping activity, an image simulator dedicated to Sentinel-2 has been set up. Thanks to this, a set of images have been simulated from various source images and combining different acquisition conditions and landscapes (mountains, deserts, cities ...).

Given disturbances have been also simulated so as to estimate the end to end performance of the processing chain.

Finally, the radiometric and geometric performances obtained by the prototype will be presented.

In particular, the geo-location performance of the level-1C products which widely fulfils the image quality requirements will be provided.

\section{INTRODUCTION}

Global Monitoring for Environment and Security (GMES) is a joint initiative of the European Commission (EC) and the European Space Agency (ESA), designed to establish a European capacity for the provision and use of operational monitoring information for environment and security applications. GMES includes a Space Component (GSC) delivering data through contributing missions and dedicated satellites: the "Sentinels". Sentinel-1 is a global SAR imaging mission, Sentinel 3 is a low resolution optical imaging and altimetry mission dedicated to land and oceans, and while Sentinel 4 and 5 are dedicated to the atmosphere monitoring respectively based on GEO and LEO satellites.

\footnotetext{
* Corresponding author.
} 
The Sentinel-2 mission [3] main objective is to provide highresolution optical imagery for the operational monitoring of land and coastal areas. As required by the user community, Sentinel-2 satellites will provide imagery for the generation of high-level operational products such as Bottom of Atmosphere reflectance maps, land-cover maps, land-change detection maps, and geophysical variables (leaf area index, leaf chlorophyll content, leaf water content...). Data from Sentinel-2 will benefit services in areas such as land management by European and national public institutes, the agricultural industry and forestry, disaster control (floods, volcanic eruptions and landslides) and humanitarian relief operations as well as surveillance of infrastructures and food security.

To maximize the reuse of European expertise gained in the area of space-borne high spatial-resolution optical sensors through missions such as the SPOT series, and to foster continuity and enhancement of operational applications, ESA and CNES have signed a cooperation agreement for the Phases C/D and E1 of the Sentinel-2 mission. This collaboration agreement is materialized at technical-level into the implementation of ESACNES technical joint teams focusing on the data-processing and image-quality areas. As such, CNES will contribute to the image products and processing definition, the prototyping of the ground processing and also the image quality commissioning phase of the Sentinel-2 spacecrafts.

This paper offers, first, an overview of the Sentinel-2 system and then, introduces the image products delivered by the ground processing. It describes in details the associated radiometric and geometric processing techniques, providing a special focus on the innovative method enhancing the accuracy of the geometric physical model.

Finally, the very promising results obtained by the processing prototyping activities will be presented and discussed. The radiometric and geometric performances will be provided as well as the associated computing time spent on the target platform.

\section{THE SENTINEL-2 MISSION AND PRODUCTS}

\subsection{Mission overview}

Sentinel-2 mission is based on a twin satellite configuration that will offer a unique combination of:

- systematic global coverage of land surfaces : from $56^{\circ}$ South to $84^{\circ}$ North,

- high revisit: every 5 days at equator under the same viewing conditions,

- high spatial resolution: $10 \mathrm{~m}, 20 \mathrm{~m}$ and $60 \mathrm{~m}$,

- multi-spectral information with 13 bands (covering visible, near infra-red and short wave infra-red),

wide field of view: $290 \mathrm{~km}$.

The satellite, design and developed by Astrium, will be deployed in a polar sun-synchronous orbit with a 14.3 orbits per day cycle. The equator will be crossed by the satellite at 10:30 am local time and the reference altitude will be $786 \mathrm{~km}$. Sentinel-2 has pointing capabilities of $\pm 20.6^{\circ}$ across the track to answer emergency requirements.

The launch of the first satellite Sentinel-2A is scheduled end2013 ; its nominal life duration is 7.25 years but the propellant is sized for 12 years.

The 13 spectral bands span from the visible and the Near InfraRed to the Short Wave Infra-Red. (fig. 1) featuring:

- 4 bands at $10 \mathrm{~m}$ : the classical blue $(490 \mathrm{~nm})$, green $(560 \mathrm{~nm})$, red $(665 \mathrm{~nm})$ and near infra red $(842 \mathrm{~nm})$ bands dedicated to land applications.
6 bands at 20m: 4 narrow bands in the vegetation red edge spectral domain $(705 \mathrm{~nm}, 740 \mathrm{~nm}, 775 \mathrm{~nm}$ and $865 \mathrm{~nm})$ and 2 SWIR large bands (1610nm and $2190 \mathrm{~nm})$ dedicated to snow/ice/cloud detection, and to vegetation moisture stress assessment.

3 bands at $60 \mathrm{~m}$ dedicated to atmospheric correction (443nm for aerosols and 940 for water vapour) and to cirrus detection $(1380 \mathrm{~nm})$.

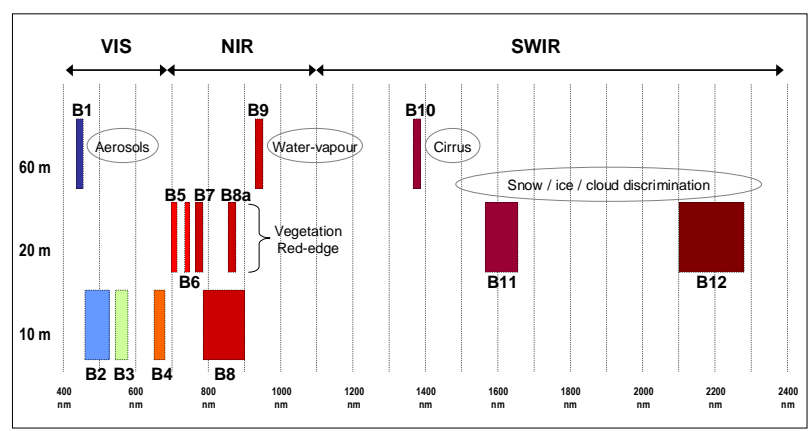

Figure 1: Spectral bands versus spatial resolution

The Multi Spectral Instrument (namely MSI) is a push-broom imager based on a TMA (Tri Mirror Anastigmatic) telescope. It is opened at F/4 and the focal length is $0.60 \mathrm{~m}$. The focal plane is split in two parts:

a Visible and Near Infra Red (VNIR) focal plane made of 12 staggered CMOS detector modules

a Short Wave Infra Red (SWIR) focal plane made of 12 staggered Mercury Cadmium Telluride (MCT) detector modules hybridized on a CMOS read-out circuit.

As well as 2 operational satellites, Sentinel-2 system includes:

- a Flight Operations Segment for satellite command, monitoring and control

- a Payload Data Ground Segment for mission planning, payload data reception, processing, archiving, quality control and dissemination

- a network of $\mathrm{S}$ bands and $\mathrm{X}$ bands stations for communication with the satellite. Nominally 4 stations should be used (e.g. Kiruna, Svalbard, Mas Palomas and Prince Albert), the final layout being consolidated in 2012.

The accurate on-board determination of the satellite position and the absolute time-stamping are supplied by a dual frequency GPS receiver. The knowledge of the satellite fine attitude is provided to the ground processing by the on board hybridization of the measurements performed by an inertial measurement unit involving gyroscopes and star trackers.

The mass memory storage capacity is 2.4 Tbits. Data are compressed using a multi-resolution compression algorithm based on a wavelet decomposition. This function operates in real time and delivers a fixed rate bit-stream with a compression ratio optimized for each spectral band in order to keep the quality of the images. The mean compression ratio is close to 3 . The mission nominal $\mathrm{X}$-band downlink data rate is $560 \mathrm{Mbps}$. In nominal mode, the satellite operates a systematic image acquisition over land. It will be possible to task for specific urgent acquisitions (near real time acquisition) where image will be available for user 3 hours maximum after sensing.

Hence, a data-strip average length will be around $6400 \mathrm{~km}$ with an average size of $177 \mathrm{~GB}$ before compression and $54 \mathrm{~GB}$ after compression. For an uncompressed square image of $290 \mathrm{~km}$ (swath) by $276 \mathrm{~km}$, the user will have to manage more than 7GB uncompressed. 
The focal plane complexity (parallax between bands and between staggered detector modules) and the huge amount of data to be processed with stringent timeliness constraints drive the processing.

\subsection{Sentinel-2 products}

One of the main features of Sentinel-2 mission is the high repetitiveness of the observations (5 days revisit). It emphasizes the potential of data time series and therefore drove the product definition: the basic Level-1 products had to be geometrically registered and radiometrically calibrated. The system product breakdown is the following:

- the Level-0 and Level-1A are system products which correspond to respectively raw compressed and uncompressed data (limited to internal calibration purposes),

- the Level-1B is the first public product: it comprises radiometric corrections (dark signal, pixels response non uniformity, crosstalk, defective pixels, restoration, and binning for $60 \mathrm{~m}$ bands); and an enhanced physical geometric model appended to the product but not applied,

- the Level-1C provides ortho-rectified top of atmosphere reflectance with a sub-pixel multi-spectral and multi-date registration; a cloud and land/water mask is associated to the product. Note that the cloud mask also provides an indication about cirrus. The ground sampling distance of Level-1C product will be $10 \mathrm{~m}, 20 \mathrm{~m}$ or $60 \mathrm{~m}$ according to the band. The final Level-1C product is tiled following a pre-defined grid of $100 \times 100 \mathrm{~km}^{2}$, based on UTM/WGS 84 reference frame,

Upper level products (e.g. Level-2) are under definition. Sentinel-2 products will also satisfy Quality Assurance for Earth Observation (QA4EO) recommendations made by CEOS. A complete cal/val and performances traceability will be given to users as well as radiometric and geometric quality index will be provided with the ancillary data of the product.

\section{IMAGE QUALITY REQUIREMENTS}

\subsection{Radiometry}

Table 2 shows the spectral bands characteristics and the required Signal to Noise Ratio (SNR) for the reference radiances (Lref) defined for the mission.

\begin{tabular}{|c|c|c|c|c|c|c|}
\hline Band & $\begin{array}{c}\text { Cent. } \\
\text { Wave } \\
\text { length } \\
(\mathrm{nm})\end{array}$ & $\begin{array}{c}\text { Band } \\
\text { width } \\
(\mathrm{nm})\end{array}$ & $\begin{array}{l}\text { Lmin } \\
\left(\mathrm{W} \cdot \mathrm{m}^{-}\right. \\
{ }^{2} \cdot \mathrm{sr}^{-} \\
\left.{ }^{1} . \mu \mathrm{m}^{-1}\right)\end{array}$ & $\begin{array}{l}\text { Lref } \\
\left(\mathrm{W} \cdot \mathrm{m}^{-}\right. \\
{ }^{2} \cdot \mathrm{sr}^{-} \\
\left.{ }^{1} \cdot \mu \mathrm{m}^{-1}\right)\end{array}$ & $\begin{array}{l}\text { Lmax } \\
\left(\mathrm{W} \cdot \mathrm{m}^{-}\right. \\
{ }^{2} \cdot \mathrm{sr}^{-} \\
\left.{ }^{1} \cdot \mu \mathrm{m}^{-1}\right)\end{array}$ & $\begin{array}{c}\text { SNR } \\
@ \\
\text { Lref }\end{array}$ \\
\hline 1 & 443 & 20 & 16 & 129 & 588 & 129 \\
\hline 2 & 490 & 65 & 11.5 & 128 & 615.5 & 154 \\
\hline 3 & 560 & 35 & 6.5 & 128 & 559 & 168 \\
\hline 4 & 665 & 30 & 3.5 & 108 & 484 & 142 \\
\hline 5 & 705 & 15 & 2.5 & 74.5 & 449.5 & 117 \\
\hline 6 & 740 & 15 & 2 & 68 & 413 & 89 \\
\hline 7 & 775 & 20 & 1.5 & 67 & 387 & 105 \\
\hline 8 & 842 & 115 & 1 & 103 & 308 & 174 \\
\hline $8 \mathrm{a}$ & 865 & 20 & 1 & 52.5 & 308 & 72 \\
\hline 9 & 940 & 20 & 0.5 & 9 & 233 & 114 \\
\hline 10 & 1380 & 30 & 0.05 & 6 & 83 & 50 \\
\hline 11 & 1610 & 90 & 0.5 & 4 & 70 & 100 \\
\hline 12 & 2190 & 180 & 0.1 & 1.5 & 24.5 & 100 \\
\hline
\end{tabular}

Table 2: Sentinel-2 spectral bands definition and Signal to noise ratio requirements for the mission
These SNR requirements, used to design MSI instrument, will provide users with images having a SNR equivalent to SPOT5 in orbit one.

The accurate knowledge of the band equivalent wavelength is very important as an error of $1 \mathrm{~nm}$ can induce a few \% errors on the reflectance, especially in the blue part (atmospheric scattering) and the red part of the spectrum (vegetation red edge). Therefore the equivalent wavelength is required to be known with an uncertainty below $1 \mathrm{~nm}$.

Getting a physical value (radiance or reflectance) from the numerical output provided by the sensor requires the knowledge of the sensor sensitivity. Any error on the absolute calibration measurement directly affects the accuracy of this physical value. It is why a maximum 5\% absolute calibration knowledge uncertainty was required for the mission, with an objective of $3 \%$. In the same way, the cross-band and multi-temporal calibration knowledge accuracy were set to respectively $3 \%$ as objective and $1 \%$.

Moreover the sensor non linearity will be known with an accuracy better than $1 \%$ and will have to be stable enough so that the detector non uniformity can be calibrated at one radiance level in flight.

Finally the system MTF (Modulation Transfer Function) including the telescope, the detector and the smearing will be better than 0.15 and lower than 0.3 at the Nyquist frequency for $10 \mathrm{~m}$ and $20 \mathrm{~m}$ bands and lower than 0.45 for $60 \mathrm{~m}$ bands.

\subsection{Geometry}

The geometric image quality requirements correspond to two types of requirements:

- programming requirements to guarantee the satellite pointing accuracy over the sites to be viewed,

- $\quad$ image quality requirements to be met by the Level-1 products using the ancillary data provided by the satellite and the data and models either calibrated in-flight or before the launch.

These requirements are summarized here under:

\begin{tabular}{|c|c|}
\hline $\begin{array}{c}\text { Geometric image quality } \\
\text { performance }\end{array}$ & Ground processing hypothesis \\
\hline $\begin{array}{c}\text { A priori accuracy of image } \\
\text { location: } 2 \mathrm{~km} \text { max }(3 \sigma)\end{array}$ & No processing \\
\hline $\begin{array}{c}\text { Accuracy of image location: } \\
20 \mathrm{~m}(3 \sigma)\end{array}$ & $\begin{array}{c}\text { After image processing } \\
\text { without control points }\end{array}$ \\
\hline $\begin{array}{c}\text { Accuracy of image location: } \\
12.5 \mathrm{~m}(3 \sigma)\end{array}$ & $\begin{array}{c}\text { After image processing with } \\
\text { control points }\end{array}$ \\
\hline $\begin{array}{c}\text { Multitemporal registration: } \\
3 \mathrm{~m}(2 \sigma) \text { for } 10 \mathrm{~m} \text { bands } \\
6 \mathrm{~m}(2 \sigma) \text { for } 20 \mathrm{~m} \text { bands } \\
18 \mathrm{~m}(2 \sigma) \text { for } 60 \mathrm{~m} \text { bands }\end{array}$ & control points \\
\hline $\begin{array}{c}\text { Multispectral registration for } \\
\text { any couple of spectral } \\
\text { bands: } 3 \mathrm{~m}(3 \sigma) \text { for } 10 \mathrm{~m} \\
\text { bands }\end{array}$ & $\begin{array}{c}\text { After image processing with } \\
\text { control points }\end{array}$ \\
$6 \mathrm{~m}(3 \sigma)$ for $20 \mathrm{~m}$ bands & \\
$18 \mathrm{~m}(3 \sigma)$ for $60 \mathrm{~m}$ bands & \\
\hline
\end{tabular}

Table 3: Geometric image quality requirements

The accuracy of the image location, without ground control points, is very good with regard to the pixel size and should satisfy most of the applications. However we will see through 
the Level-1 processing description that most of Sentinel-2 products will take benefits from GCP and satisfy a $12.5 \mathrm{~m}$ maximum geolocation accuracy error

The multi-temporal registration performances require a knowledge of the non linear temporal component of the attitude better than $0.1 \mu \mathrm{rad} / \mathrm{s}$; we assume here that the ground processing will be able to calibrate any linear temporal variation of the attitude using ground control points.

\section{THE LEVEL-1 PROCESSING}

The Sentinel-2 Level-1C products will take advantage of the stringent image quality requirements and provide users with ortho-rectified images representing Top of Atmosphere reflectance.

The following figure offers an overview of the Level-1 processing which combines radiometric and geometric corrections.

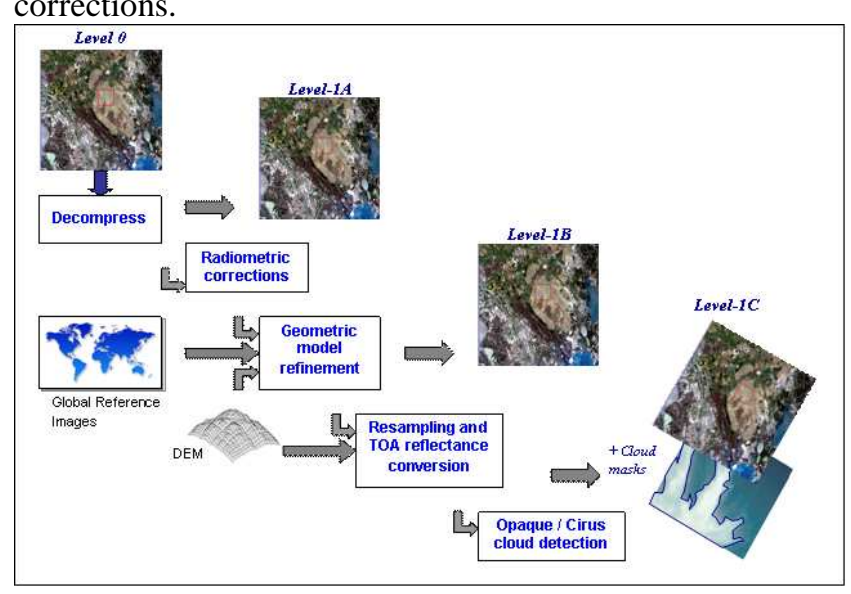

Figure 4: Level-1 processing workflow

\subsection{Radiometric corrections}

Following the decompression of the image source packets transmitted by the MSI and extraction of ancillary information, the radiometric corrections deal with:

- Normalization: dark signal and detector relative sensitivity correction

- SWIR pixels arrangement: for the SWIR bands, the detector module is made of 3 lines for B10 and 4 lines for B11 and B12. So as to benefit from the pixels having the best SNR for the acquisition, for each column, 1 pixel over 3 lines is selected for B10 and 2 successive pixels over 4 lines are selected for B11 and B12 (bands working in TDI (Time Delay and Integration) mode). At ground level, a simple pixels re-arrangement is performed thanks to the earth rotation compensation.

- Crosstalk: despite a very demanding cross-talk specification, the high radiance dynamics specified for Sentinel-2 may induce defects that need to be corrected. This phenomenon, mainly electronics, will be completely characterized and modeled before the launch.

- Defective pixels: for less than $0.1 \%$ of the pixels (specification), a column interpolation may have to be done,

- Image restoration combining deconvolution to correct the overall MTF (frequential processing) and denoising based on a wavelet processing [2].
Binning: for $60 \mathrm{~m}$ bands as the spatial resolution acquired across the track is $20 \mathrm{~m}$.

\subsection{Geometric model refinement}

The goal of the geometric correction is to perform the temporal and spectral registration of all the images taken over any target. To reach this objective, the physical geometric model, which associates a viewing direction to any pixel, has to be refined (fig. 5) according to an absolute reference.

This physical geometric model combines position, attitude and time-stamp information, transformation matrices between different reference frames: satellite, instrument, focal planes and detectors, and for each elementary detector a viewing direction. Then, the processing chain takes advantage of a Global Reference Image database, perfectly geo-referenced, to correct the physical geometric model of each image taken.

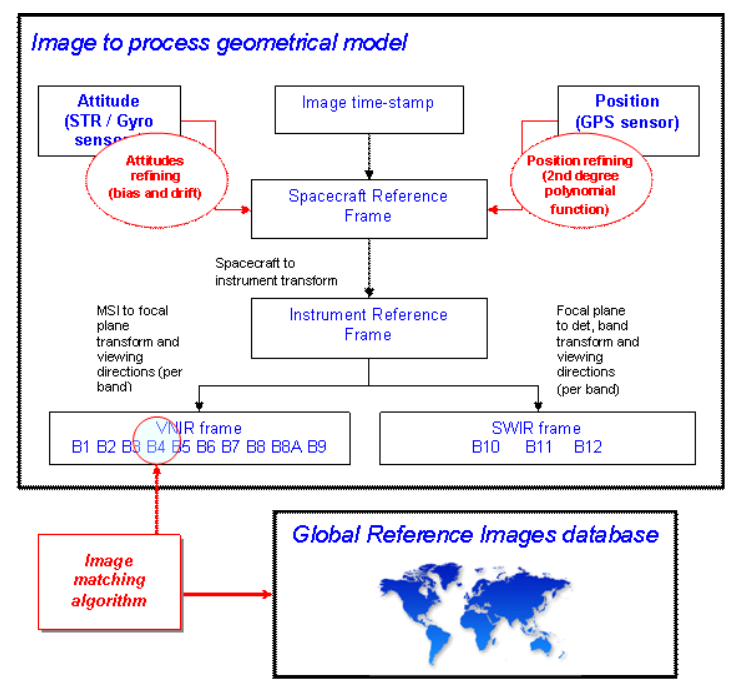

Figure 5: Sentinel-2 physical geometric model refinement based on automatic correlation with reference data

The processing is based on an automatic image matching process [4] which provides accurate ground control points between a given band of the image to refine and a reference image, allowing to dynamically calibrate the viewing model and so to attitude and position profiles [5].

Because of the parallax between odd and even detector modules and between bands, the registration is sensitive to the Digital Elevation Model (DEM) used for the processing. A DTED1class DEM is necessary to reach the required performances. The refined geometrical model is then propagated to all the bands.

The geometric model refinement also implements an automatic registration between VNIR and SWIR focal planes based on multi-spectral correlation (based on similar method). This complementary refinement could be used during commissioning phase.

\subsection{Resampling, TOA reflectance conversion and Cloud detection}

Once the geometrical model of the image is refined, the final ortho-rectification is performed using an optimized resampling process combining:

a tiling process, partitioning the final images in several tiles according to the cartographic pre-defined grid, 
- a geometric process computes the inverse grid that gives for each point of the output image its location in the focal plane,

a radiometric process computes for each point of the grid its radiometry using Splines interpolation functions (in radiance),

- a final operation converts the radiance into the reflectance (knowing the sensor sensitivity). This operation is done on the fly at pixel level.

Finally a cloud (opaque/cirrus) and land/water mask is computed based on spectral criteria:

- clouds have a high reflectance in the blue (443 $\mathrm{nm}$ or 490 $\mathrm{nm})$ and SWIR (1380 $\mathrm{nm}$ or $2190 \mathrm{~nm}$ ) bands

cirrus have a low reflectance in the blue bands and a high reflectance at $1380 \mathrm{~nm}$

water has a low reflectance in the Near Infra Red (865nm) and SWIR $(1380 \mathrm{~nm}$ or $2190 \mathrm{~nm})$ bands

\subsection{A key element, the Global Reference Images database}

A key improvement in the overall process is the usage of the Global Reference Images database. This database will be made of Sentinel-2 mono-spectral images whose geometrical model will be refined. It will be initialized during the commissioning phase.

Indeed, during this phase, a set of cloud-free images over a given area (likely Europe) will be processed with a dedicated method, already used for SPOT5-HRS [1], based on a large space-triangulation (bundle block adjustment on a large area), using tie points between the different images and GCP in order to improve jointly the geometric models of images.

The process takes also advantage of the overlap between Sentinel-2 orbits. In a second stage, the database will be improved by adding new images, for example to cover areas which would be cloudy in the first stage. The location of the new images is then corrected so as to be consistent with the former database, which will be considered as a fixed reference. In the same way, the database is globally extendable, as the images become available all around the world, the geolocation of the new images being corrected with respect to the first reference database.

It is important to underline, that any image with a refined geometric model can then become a new reference. It allows updating the reference according to the landscape seasonal evolutions.

\section{PROTOTYPING ACTIVITIES}

In the frame of the ESA-CNES cooperation for the Sentinel-2 program, ESA has delegated to CNES the technical definition of the Sentinel-2 Level-0/1A/1B/1C Ground Prototype Processor (GPP) as well as the follow-up of its industrial development. The prototype will be used for the validation of the performance of the processing of MSI products.

It will also be used to process the Sentinel-2 MSI Mission data to Level-0/1A/1B/1C during the commissioning phase and to verify the proper functioning of the operational processor to be implemented by ESA within the Payload Data Ground Segment (PDGS).

Based on the integration of existing CNES image processing libraries (for the generic parts such as geometric modeling and refinement, image restoration) together with new developments for the specific Sentinel-2 parts (crosstalk corrections, normalization,...), the prototype is currently being integrated by a European industrial Consortium led by Advanced Computer
Systems (ACS) S.p.A. as prime contractor and involving MAGELLIUM and DLR as subcontractors.

Furthermore, an image simulator dedicated to Sentinel-2 has been set up at CNES in order to perform independent validation of the prototyping activity. Inherited from former CNES earth observation missions, this simulator consists of two main parts:

- a geometrical simulation of the acquisition going from a high resolution source image of the landscape to the 12detectors image acquired, taking into account the overall MTF),

a radiometric simulation (radiometric defects, noise, multi spectral pattern injection, etc...).

Thanks to this, a set of Sentinel-2 images have been simulated from various source images and combining different acquisition conditions and landscapes (mountains, deserts, cities ...).
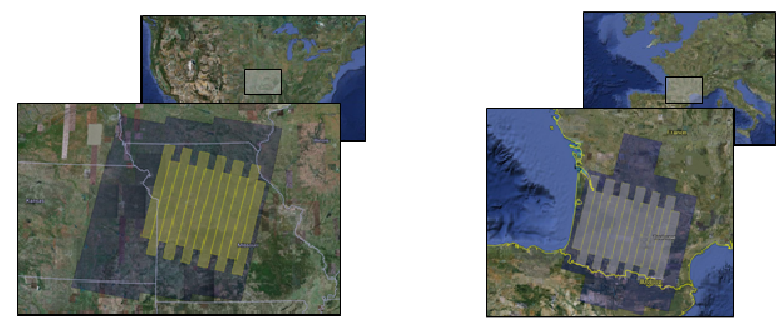

Figure 6 : Footprints of a source images (blue) and associated S2 simulated images (yellow) cases over Missouri (US) and Midi-Pyrenees (FR) areas

Moreover, given disturbances have been simulated so as to estimate the end to end performance of the processing chain: geometric level: injection of orbital restitution error (linear drift (time dependant) from $5 \mathrm{~m}$ to $10 \mathrm{~m}$ in absolute), radiometric level: insertion of defective pixels, MTF modeling, noise addition, optical and electronic crosstalk contributions, detector cubic response...

The simulated test data are then used to validate the prototype. The cross-validation campaign is still on-going.

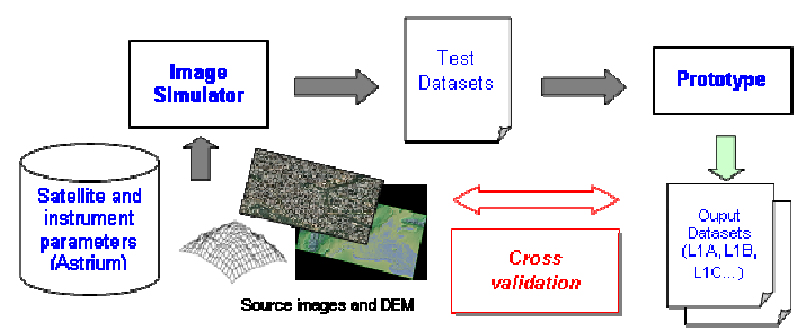

Figure 7: Simulator and prototype cross-validation

The following pictures shows intermediary results obtained through the prototype during the radiometric corrections stage.

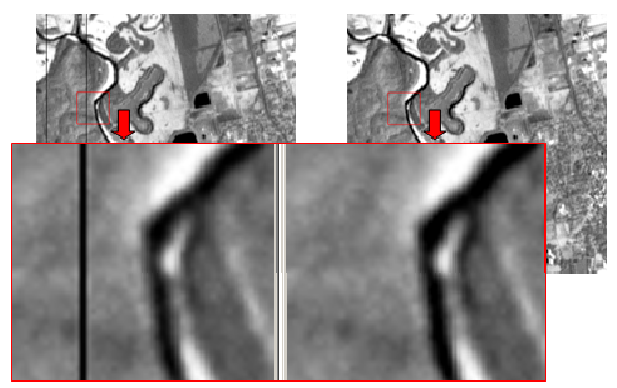


Figure 8: Radiometric corrections - Detector interpolation

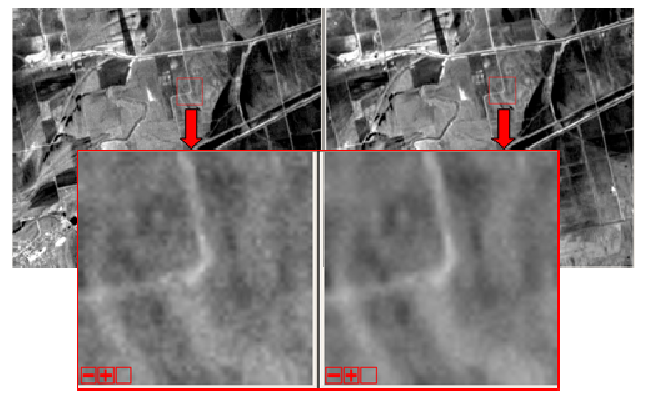

Figure 9: Radiometric corrections - B2 band before and after de-noising

Finally, the geo-location performance of the Level-1C orthoimage is systematically assessed by computing the residual misregistration with the initial ortho-image.

The following figure represents an example of the cumulative histogram on the residual (in $10 \mathrm{~m}$ pixels) between both images measured by automatic correlation. It shows that the residual error is less than $\sim 1 / 4$ pixels for $90 \%$ of the points.
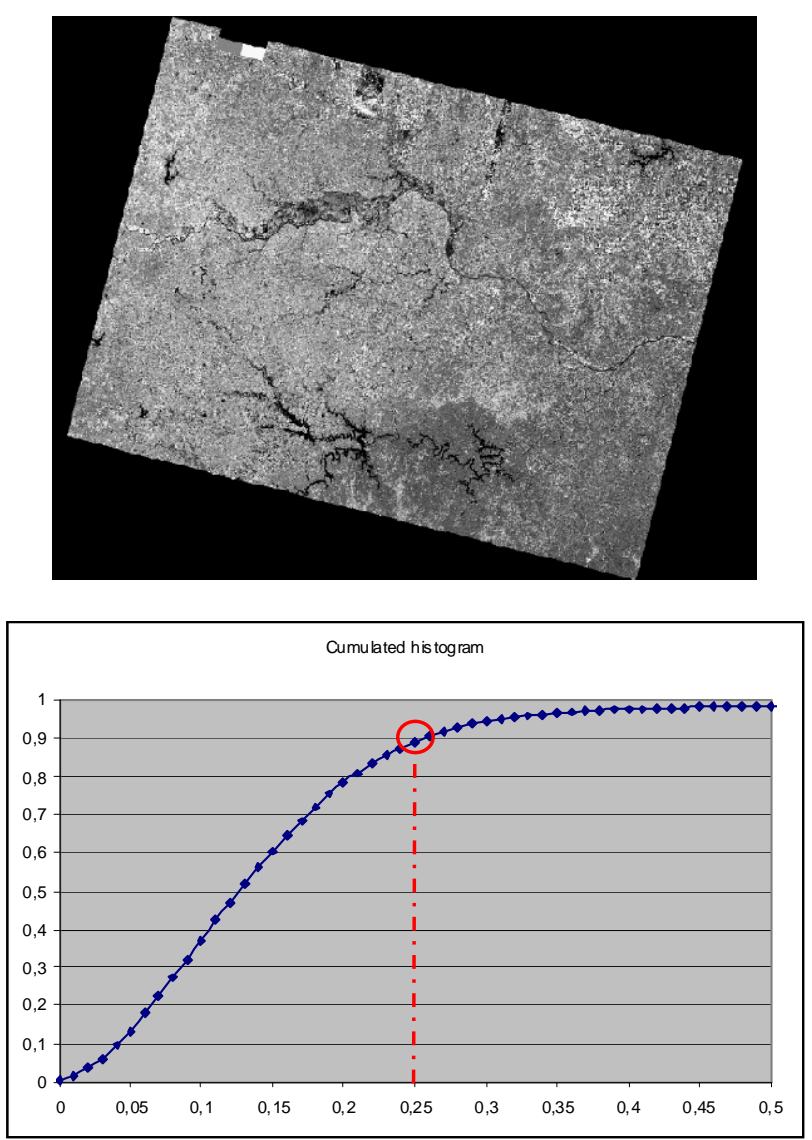

Figure 10: Level-1C Ortho-image and cumulative histogram on registration residuals (in pixels)

These first results are very promising and must be consolidated. In particular, the processing parameters are being adjusted for the geometrical part and the validation campaign will confirm these results $\mathrm{m}$ on the entire validation data set. The computing time estimations are in-line with the expectations and less than
$45 \mathrm{~min}$ will be needed to process up to Level-1C a 290x290 $\mathrm{km}^{2}$ image with the prototype.

\section{CONCLUSIONS}

As described, Sentinel2 image processing is very complex (especially for geometric corrections) and shall handle a huge amount of data. It has been therefore very challenging to design, in a short time frame, an ambitious Ground Prototype Processor that will be in charge of generating the Level-1 products during the commissioning phase, with stringent timeliness requirements (more than 700 products to generate).

The first results are very encouraging in term of image quality performance as well as in term of computing performance. This has been made possible thanks to an important re-use of the existing optimized image processing libraries, legacy from previous CNES missions (SPOT, Pleiades-HR, ...). As such, this project is an example of successful and efficient cooperation between European space agencies in the specific field of optical image remote sensing.

\section{References}

[1] S. Airault, Reference3D accuracy and quality process: First assessment and perspectives, ISPRS Workshop Oct. 2003, Hanover.

[2] P. Deherete, B. Rouge, Image de-blurring and application to SPOT5 THR satellite imaging, IGARSS '03, Toulouse

[3] F. Gascon, M. Berger, Sentinel-2 Mission Requirements Document, http://esamultimedia.esa.int/docs/GMES/GMES_Sentinel2_MR D_issue_2_0_update.pdf

[4] J. Le Moigne, et al., Image Registration for Remote Sensing, Cambridge University Press, (2011), Section 19 (http://www.cambridge.org/us/9780521516112).

[5] S. Baillarin, P. Gigord, O. Hagolle, Automatic Registration of Optical Images, a Stake for Future Missions: Application to Ortho-Rectification, Time Series and Mosaic Products, IGARSS 2008

\section{Acknowledgements}

We would like to thank all our Sentinel-2 colleagues at ESA, CNES, Astrium, ACS, MAGELLIUM and DLR. We are grateful to C. Zelli, E. Hillairet, T. Storsch and their teams for their highly valuable work on the Sentinel2 Ground Processor Prototype. 